Article

\title{
Fault Isolability Analysis and Optimal Sensor Placement for Fault Diagnosis in Smart Buildings
}

\author{
Max Emil S. Trothe, Hamid Reza Shaker* $*$, Muhyiddine Jradi $@$ and Krzysztof Arendt \\ Center for Energy Informatics, The Maersk Mc Kinney Moller Institute, University of Southern Denmark, \\ 5230 Odense, Denmark; mtrot14@student.sdu.dk (M.E.S.T.); mjr@mmmi.sdu.dk (M.J.); \\ krza@mmmi.sdu.dk (K.A.) \\ * Correspondence: hrsh@mmmi.sdu.dk
}

Received: 27 March 2019; Accepted: 25 April 2019; Published: 26 April 2019

\begin{abstract}
Faults and anomalies in buildings are among the main causes of building energy waste and occupant discomfort. An effective automatic fault detection and diagnosis (FDD) process in buildings can therefore save a significant amount of energy and improve the comfort level. Fault diagnosability analysis and an optimal FDD-oriented sensor placement are prerequisites for effective, efficient and successful diagnostics. This paper addresses the problem of fault diagnosability for smart buildings. The method used in the paper is a model-based technique which uses Dulmage-Mendelsohn decomposition. To the best of our knowledge, this is the first time that this method is used for applications in smart buildings. First a dynamic model for a zone in a real-case building is developed in which faults are also introduced. Then fault diagnosability is investigated by analyzing the fault isolability of the model. Based on the investigation, it was concluded that not all the faults in the model are diagnosable. Then an approach for placing new sensors is implemented. It is observed that for two test scenarios, placing additional sensors in the model leads to full diagnosability. Since sensors placement is key for an effective FDD process, the optimal placement of such sensors is also studied in this work. A case study of campus building OU44 at the University of Southern Denmark is considered. The results show that as the system gets more complicated by introducing more faults, additional sensors should be added to achieve full diagnosability.
\end{abstract}

Keywords: fault diagnosability; sensor placement; smart buildings; Dulmage-Mendelsohn decomposition

\section{Introduction}

Buildings in the EU account for $40 \%$ of the total energy consumption [1]. Thus, the building sector is considered to be the largest single contributor to the energy consumption in the world [2]. Also, the building sector is responsible for $36 \%$ of the total $\mathrm{CO}_{2}$ emissions in the $\mathrm{EU}$, which makes the building sector the highest contributor to greenhouse gas emissions [1,2]. In buildings, HVAC systems are especially important as they are energy intensive and complex in their operation patterns [3]. Studies show that in developed countries, HVAC systems account for up to $50 \%$ of the energy use in buildings [4]. However, a high proportion of the electricity consumption in the HVAC systems comes from energy waste and is caused by inefficient operation of the system. Studies have estimated that up to $30 \%$ of the energy consumption within commercial buildings can be attributed to faults which are caused by systems working non-optimally [5]. Further, predictions indicate that the energy consumption in the EU will increase by 50\% during the next 15 years $[4,6]$. Therefore, with the energy consumption set to increase, there is an urgent need to investigate methods that can detect and diagnose faults within buildings i. e. Fault detection and Diagnosis (FDD) methods [7]. Another study states that some of the most influential faults in small commercial buildings are due to building operation. 
By considering the annual energy impact of a fault, the study listed incorrect HVAC on/off modes, inappropriate set points and zone temperature sensor bias as some of the most common causes of energy wastage [8]. Further, the annual financial impact of a fault was investigated. In the United States, incorrect HVAC on/off modes cost some $\$ 920$ million per year, while inappropriate operation set points contribute another $\$ 492$ million per year [8]. In order to overcome faults in HVAC systems, the ability to detect faults and diagnose their causes is fundamental. Here, automated fault detection and diagnostics (FDD) approaches are used to deal with the problem. Sorrentino et al. [9] proposed a model-based method for FDD that uses a key performance indicator to detect inefficient management of cooling systems. The method is applied for building applications where it can provide knowledge about the cooling strategies in order to improve energy performance of the building. Further, the method has been pointed out as a potential method within fault diagnosis [9]. In another study, the authors discuss a diagnostic technique that is able to improve fault isolability [10]. Within fault diagnosis, the ability to isolate faults is considered to be vital. The suggested diagnostic technique has been tested on fuel cells, where residual generators have been used. Here, the residual generators react when a component is under faulty state. Thereby, the residual generators provide knowledge about the fault appearing in different components of the fuel cell. Even though FDD has been an active research area over the last few decades, there is a lack of automated FDD approaches that can be used for HVAC systems within buildings [11]. Further, the state-of-art FDD methods lack the ability to predict the overall impacts of the faults [12]. A recent review paper [13] give a more detailed overview of the state-of-art in FDD for the building application. Taking into account that HVAC systems contribute to a large part of the energy consumption of buildings and there is a lack of FDD methods for building applications, there is an urgent need to investigate new FDD methods that can be applied for building applications.

This study proposes an FDD bottom-up method that is based on a dynamic building model. The method was implemented and tested to diagnose faults within the HVAC system in buildings. The method can be applied for entire HVAC systems, which means that it can take into account the heating, ventilation and air conditioning within a building. However, in order to simplify the method, this paper only looks into the ventilation system of a zone. Thus, the method was deployed considering a zone of the OU44 building located at the University of Southern Denmark Campus, in Odense (Denmark).

Frisk et al. [14] proposed a method that can provide knowledge about a fault detectability and isolability based on a Dulmage-Mendelnsohn decomposition $[14,15]$. Fault isolability describes the ability for a fault to be isolated from other faults within the model. Thus, a single fault can cause other faults to occur if the faults are not isolated. As the complexity has increased in modern technical systems, the ability to isolate faults from each other has become increasingly important [7]. In this paper, the Dulmage-Mendelnshon decomposition is implemented into the ventilation system of OU44 building. To the best of knowledge, this is the first time that this method is applied for building applications. A dynamic model for a zone in the building is developed in which faults are also introduced [16].

The study is divided into two scenarios. The first scenario considers three sensors measuring the temperatures. For each of the sensors, a fault of an unknown size is introduced. For the second scenario, the ventilation damper position and the radiator valve position are taken into account. Here, an unknown percentage error is added to each of the valves. The percentage error lies in the interval between 0 and $100 \%$.

\section{Methodology}

Within the area of FDD, there exist several different fault detection and diagnosis methods that seeks to detect a fault and determine the influence and consequences of the fault. Basically, the methods can be divided into three groups. There are qualitative model-based methods, quantitative model-based methods and process history-based methods [11]. Qualitative model-based methods are usually based on rule-based models, while quantitative model-based methods use the understanding of the physical 
relationships between the different parameters and components within the system to construct either detailed or simplified physical models. Process history-based methods concentrate on either black-box or gray-box models. The process history-based methods are data driven and rely on knowledge and measurements on inputs and outputs of the model. Each FDD method has strength and weaknesses corresponding to different usage.

For this study, a quantitative model-based method is used. The study uses a detailed physical model that considers relationships between various temperatures in a zone. In order for the physical model to fit the requirements of the Fault Diagnosis Toolbox (FDT) which has been used in this study, it has been necessary to make some assumptions regarding the model inputs. Therefore, the method uses a combination of detailed- and simplified physical models.

In order to conduct the fault diagnosability analysis of the model, a Dulmage-Mendelnsohn decomposition is used. The Dulmage-Mendelnsohn decomposition provides knowledge about the different parts of the model and the detectability and isolability of the faults within the model. Preliminary knowledge about the faults isolability is also revealed in the Dulmage-Mendelsohn decomposition. Based on the physical model, the paper looks into two scenarios where a different number of faults are introduced. For each scenario, an isolability analysis is conducted. The sensor placement analysis is made in which it is studied where additional sensors could be placed to increase both the fault detectability and isolability $[15,17]$. Then sensors are added, and the fault detectability is investigated in the Dulmage-Mendelsohn decomposition. Based on the faults location in the Dulmage-Mendelsohn decomposition, it can be determined whether a fault is detectable or not. The improved detectability of the faults should be recognized as the faults enter the overdetermined part of the model. The fault isolability is investigated in the isolability matrices, where the relationships between the different faults can be seen. The methods are applied to the study by using a MatLab-driven Fault Diagnosis Toolbox (FDT) [14].

In order to use the features of the FDT, it is required that the model does not contain any underdetermined parts. However, as the considered model contains a lot of unknown variables, parts of the model are underdetermined. In such a case, the sensor placement analysis cannot be properly conducted, as the number of sensor sets ensuring, e.g., fully isolability becomes too large for the toolbox to handle. In order to avoid the underdetermined parts of the model, there are two options: (1) to neglect some variables, or (2) to assume that they are constants. This study uses option (2) to assess two different scenarios, so in order to avoid parts of the model becoming underdetermined, some variables have been made constant.

\section{Modeling Approach}

In order to illustrate the FDD-method applied in this study, a dynamic model for a zone in an actual building is developed. Here, the study uses a model developed by Arendt et al. [16]. Initially, the zone is represented in a state-space model which is obtained from the consideration of RC thermal networks within the zone of a building [16]. The state-space model takes into account sensors that measure temperatures on the external and internal sides of walls, as well as the outdoor and room temperature. In order to be able to introduce faults to the model, the model must be rewritten from its state-space form to a dynamic model.

\subsection{Case Study Building}

In general, there exists two kinds of model-based approaches related to FDD. These are the top-down approach, in which a whole building is investigated and analyzed with respect to fault detecting and diagnosis. The second method is the bottom-up method in which subsystems and/or zones of the building are analyzed $[18,19]$. In this study the bottom-up approach is used, as a zone of the OU44 building located at the University of Southern Denmark Campus Odense is used as a case study. The room serves as a classroom that is mainly used for teaching, and the occupancy pattern is therefore quite consistent. The room is equipped with a large number of sensors including multiple 
temperature sensors, ventilation damper opening position and heater valve position. The building is shown in Figure 1.

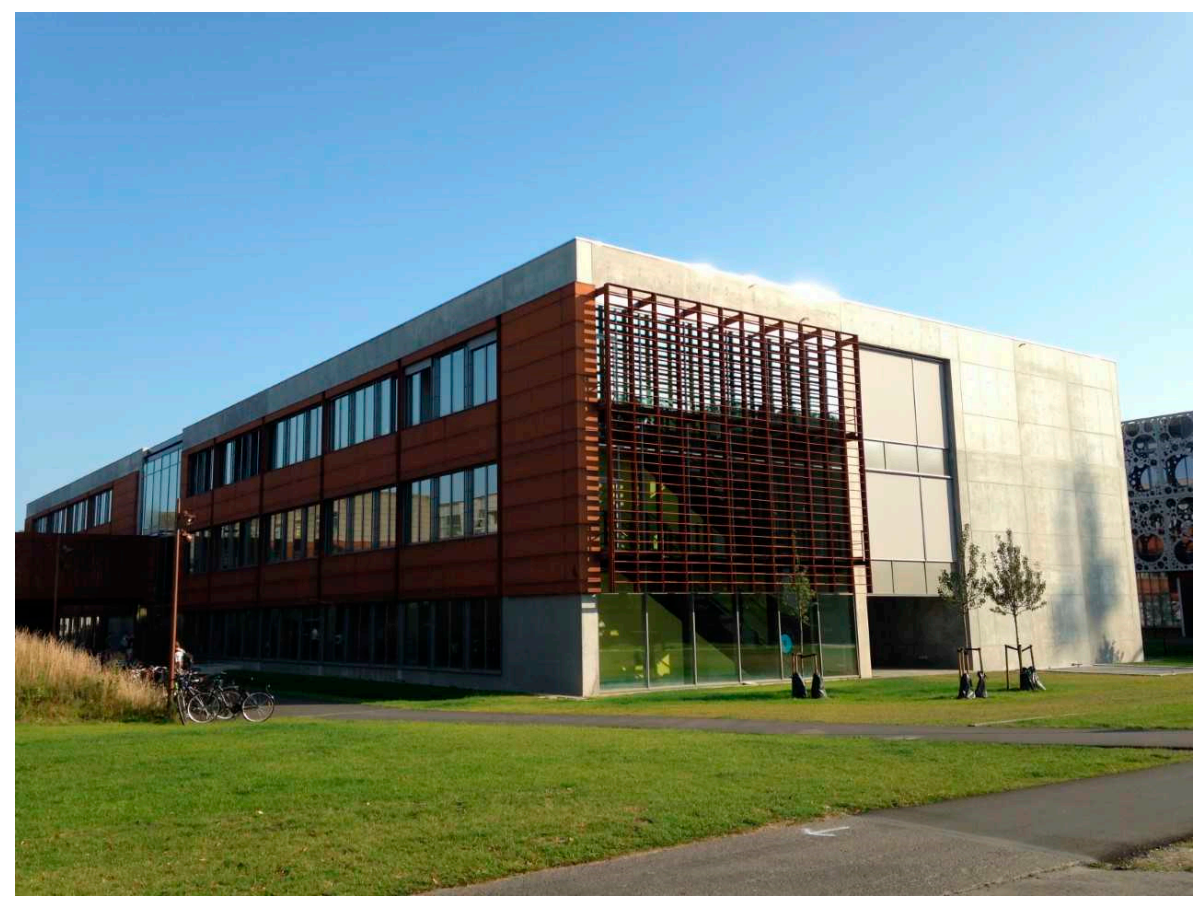

Figure 1. The OU44 building located at the University of Southern Denmark Campus Odense seen from southwest.

\subsection{Dynamic Model}

As mentioned, the study uses the model developed by Arendt et al. [16]. Initially, the model is expressed in state-space form, but is now rewritten to a dynamic model.

The time derivatives of the model are described in the following equations:

$$
\begin{gathered}
\dot{T}_{r}=\left(-\left(\frac{1}{R_{i}}+\frac{1}{R_{e 1}}\right) \frac{1}{C_{r}}\right) T_{r}+\left(\frac{1}{R_{i} C_{r}}\right) T_{i}+\left(\frac{1}{R_{e 1} C_{r}}\right) T_{e}+\left(\frac{f_{\text {sol }}}{C_{r}}\right) H_{g l o}+\left(\frac{Q_{r a d}}{C_{r}}\right) v_{p o s}+\left(\frac{1}{C_{r}}\right) q_{v e}+\left(\frac{1}{C_{r}}\right) q_{o c c} \\
\dot{T}_{i}=\left(\frac{1}{R_{i} C_{i}}\right) T_{r}-\left(\frac{1}{R_{i} C_{i}}\right) T_{i} \\
\dot{T}_{e}=\left(\frac{1}{R_{e 1} C e}\right) T_{r}-\left(\left(\frac{1}{R_{e 1}}+\frac{1}{R_{e 2}}\right) \frac{1}{C_{e}}\right) T_{e}+\left(\frac{R_{e 2}}{C_{e}}\right) T_{\text {out }}
\end{gathered}
$$

where $T_{r}$ is the room temperature $\left({ }^{\circ} \mathrm{C}\right), T_{i}$ is the internal thermal mass temperature $\left({ }^{\circ} \mathrm{C}\right)$, and $T_{e}$ is the external thermal mass temperature $\left({ }^{\circ} \mathrm{C}\right)$, and $\dot{T}_{r}, \dot{T}_{i}, \dot{T}_{e}$ are the respective time derivatives. $R_{i}, R_{e 1}$ and $R_{e 2}$ represent the internal and external wall resistance $(\mathrm{K} / \mathrm{W})$, respectively. $C_{r}$ is the thermal capacitance $(\mathrm{J} / \mathrm{K})$ for the room, while $C_{i}$ and $C_{e}$ is the internal and external thermal mass capacitance $(\mathrm{J} / \mathrm{K})$, respectively.

The outdoor temperature $T_{\text {out }}\left({ }^{\circ} \mathrm{C}\right)$, the horizontal global radiation $H_{g l o}(\mathrm{~W})$, the radiator valve position $v_{p o s}(\%)$, the ventilation heat gain $q_{v e}(\mathrm{~W})$, and the occupancy heat gain $q_{o c c}(\mathrm{~W})$ are all inputs to the model. Also, the model contains parameters as the solar coefficient $f_{s o l}$ and the maximum radiator power $Q_{\text {rad }}(\mathrm{W})$.

Next, the output functions-where it is possible to introduce faults-are described. Here, $y_{T r}$ is the sensors measurement of $T_{r}$. The output functions are given as:

$$
y_{T r}=T_{r}
$$




$$
\begin{gathered}
y_{T i}=T_{i} \\
y_{T e}=T_{e} \\
y_{q_{v e}}=q_{v e}
\end{gathered}
$$

where the ventilation heat gain, $q_{v e}$, is expressed as follows:

$$
q_{v e}=\left(d_{p o s} \cdot V_{\max }\right)\left(T_{v e}-T_{r}\right)
$$

Here, $d_{p o s}$ is the ventilation damper position (\%), $V_{\max }$ is the maximum airflow rate into the room when damper is fully open $\left(\mathrm{m}^{3} / \mathrm{h}\right)$, and $T_{v e}$ is ventilation air temperature $\left({ }^{\circ} \mathrm{C}\right)$.

In order for the FDT to work, the differential constraints of the model must also be defined. These are as follows:

$$
\begin{aligned}
& \dot{T}_{r}=\frac{d}{d t} T_{r} \\
& \dot{T}_{i}=\frac{d}{d t} T_{i} \\
& \dot{T}_{e}=\frac{d}{d t} T_{e}
\end{aligned}
$$

As the model has been expressed, it is possible to introduce the faults. This study focuses on two kinds of faults. The first fault is sensor faults which makes the sensors that measure the temperature at different locations become faulty. The second fault is a percentage fault in the opening of the radiator valve position and the damper position.

\subsubsection{Scenario 1}

In scenario 1 , only the sensor faults are to be considered. This is done by assuming the $v_{p o s}, d_{p o s}$, $V_{\max }$, and $H_{g l o}$ to be constant. The sensor faults $f_{T_{r}}, f_{T_{i}}, f_{T_{e}}$ are added to the respective temperature measurements:

$$
\begin{aligned}
& y_{T r}=T_{r}+f_{T_{r}} \\
& y_{T i}=T_{i}+f_{T_{i}} \\
& y_{T e}=T_{e}+f_{T_{e}}
\end{aligned}
$$

The faults cause the corresponding sensors to report faulty temperature measurements. The faults can have profiles that correspond to a ramp or parabola input. If the faults are expressed as a ramp or parabola input, the measured temperature will be different from the actual temperature. Otherwise, the faults can have sinusoidal input characteristic. Here, the fault value added to the measured temperature will change over time. All type of fault characteristics will cause the measure temperature to be either higher or lower than the actual temperature.

\subsubsection{Scenario 2}

Scenario 2 takes into account the positions of ventilation damper and radiator valves. In order to do so, the $v_{p o s}$ and $d_{p o s}$ are no longer assumed to be constant. Instead, $q_{o c c}$ is assumed to be constant as well as $T_{v e}$. This is done in order to avoid any underdetermined parts in the model.

The faults $f_{v_{p o s}}$ and $f_{d_{p o s}}$ are introduced to $v_{p o s}$ and $d_{p o s}$, respectively:

$$
\begin{aligned}
& v_{p o s} \cdot\left(1 \pm f_{v_{p o s}}\right) \\
& d_{p o s} \cdot\left(1 \pm f_{d_{p o s}}\right)
\end{aligned}
$$

Both $f_{v_{p o s}}$ and $f_{d_{p o s}}$ are percentage faults and can change from 0 to $100 \%$. Further, they are assumed to be time-varying that are influenced by temperature changes throughout the day. The introduced 
faults to the valve and damper positions cause them to deviate from the setpoint position. Faulty $v_{\text {pos }}$ and $d_{p o s}$ affect the room temperature through Equation (1).

\section{Results and Discussions}

\subsection{Dulmage-Mendelnsohn Decomposition}

For each of the two scenarios, a fault diagnosability analysis is conducted. This is done by using the described methodology through the FDT.

For the fault diagnosis analysis, a Dulmage-Mendelsohn decomposition is used. Figure 2 shows the Dulmage-Mendelsohn decomposition of scenario 1. The Dulmage-Mendelsohn decomposition allows the identification of three parts within the model: (1) an overdetermined part, (2) a just-determined part, and (3) an underdetermined part. The listed parts are functions of the number of equations and unknown variables introduced [20]. The overdetermined part introduces more equations than unknown variables, while the under-determined part introduces more unknown variables than equations. The just-determined part of the model introduces the same number of unknowns as equations. In terms of fault diagnosis, the overdetermined part of a model is very interesting, since the overdetermined part is the part with redundancy [21]. Faults that appear in the overdetermined part of the model are structurally detectable and can be used for further fault diagnosis and sensor placement analyses.

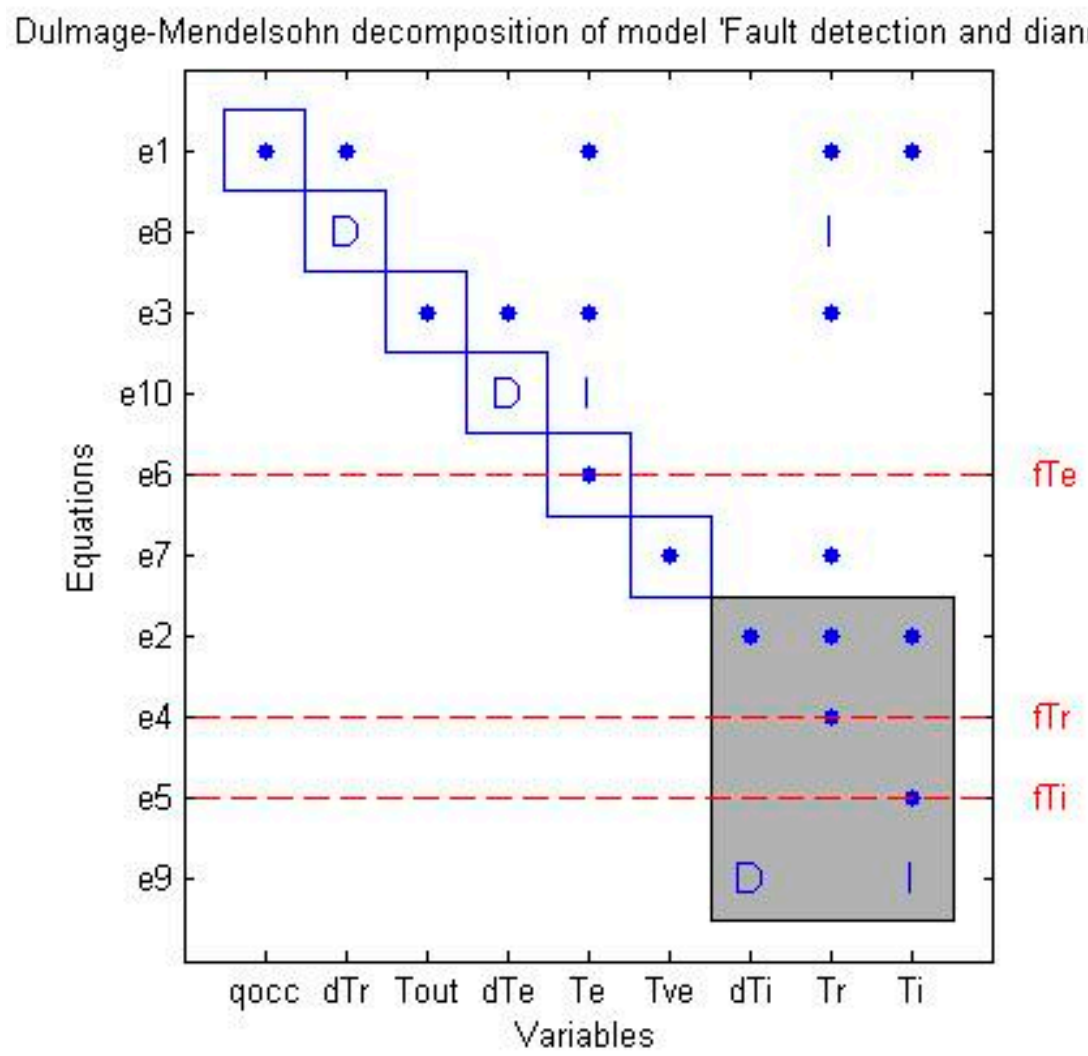

Figure 2. A Dulmage-Mendelsohn decomposition of scenario1 showing the faults location within the model.

Another result output from the FDT is the degree of redundancy. In the Dulmage-Mendelnsohn decomposition, redundancy is found in the overdetermined parts of the model. The redundancy within the model is interesting with respect to fault diagnosis and the design of residual generators [21]. Thus, residual generators are designed by using equations that contain redundancy. Therefore, it is preferable 
to have a high degree of redundancy within the system. For scenario 1 and 2, the redundancy degree is 1 , which is low if the model should be used to residual generator design.

In general, the Dulmage-Mendelsohn decomposition is a good approach for the preliminary analysis of the model. Mainly, the Dulmage-Mendelsohn decomposition is useful to gather information about underdetermined parts and about the detectability of the faults. However, when it comes to the isolability analysis, the Dulmage-Mendelsohn decomposition is not enough. In order to conduct a detailed isolability analysis, it is necessary to look into the isolability matrices.

\subsection{Isolability Analysis}

Isolability analysis of the scenarios is conducted by performing a fault isolability matrix. Here, the faults relationship to each other is studied. In the fault isolability matrix, each blue dot is a relation between two faults. The $\mathrm{x}$ axis and $\mathrm{y}$ axis represent different faults occurring. Here, a blue dot in position $(i, j)$ indicates, that fault $i$ will occur if fault $j$ is present [20]. Based on this, the isolability of the faults can be investigated.

The result of the analysis is an isolability matrix that can be seen in Figures 3 and 4 for scenarios 1 and 2, respectively. It can be seen that $f_{T r}$ and $f_{T i}$ does not occur if $f_{T e}$ is a fault. However, if any of $f_{T r}$ or $f_{T i}$ is a fault, all faults will be present. Thereby, one fault can cause in total three faults to occur at the same time due to bad isolability.

The fault isolability can be increased by adding additional sensors to the system. Additional sensors can both increase fault detectability and fault isolability [15]. Extra sensors can be added by using an experimental approach. Here, sensors can be repeatedly added to the system and it can be seen how each added sensor influences the isolability. This is very useful to see the outcome of adding a specific sensor to the system. Also, the FDT has the opportunity of determining the minimum number of additional sensors required to ensure either full detectability or full isolability [21]. The FDT uses knowledge about how each added sensor could affect the isolability. Thus, it can suggest exactly where sensors should be added. Likewise, the FDT can provide knowledge on which sensor locations do not improve the isolability. This feature has been used in both scenarios 1 and 2 .

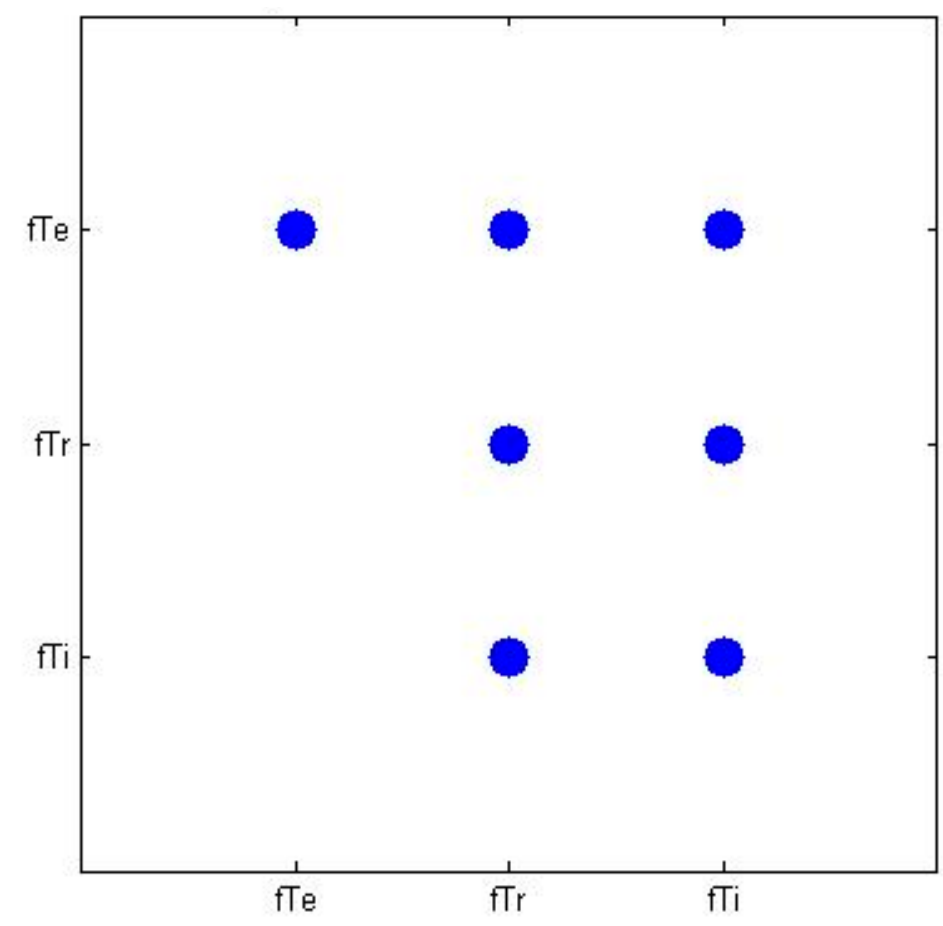

Figure 3. Fault isolability matrix for scenario 1, where the relationship between the faults can be seen. The faults $f_{T r}$ and $f_{T i}$ does not occur if $f_{T e}$ is a fault. However, if the faults $f_{T r}$ or $f_{T i}$ occurs, all faults within scenario 1 will be present as they are isolated from each other. 


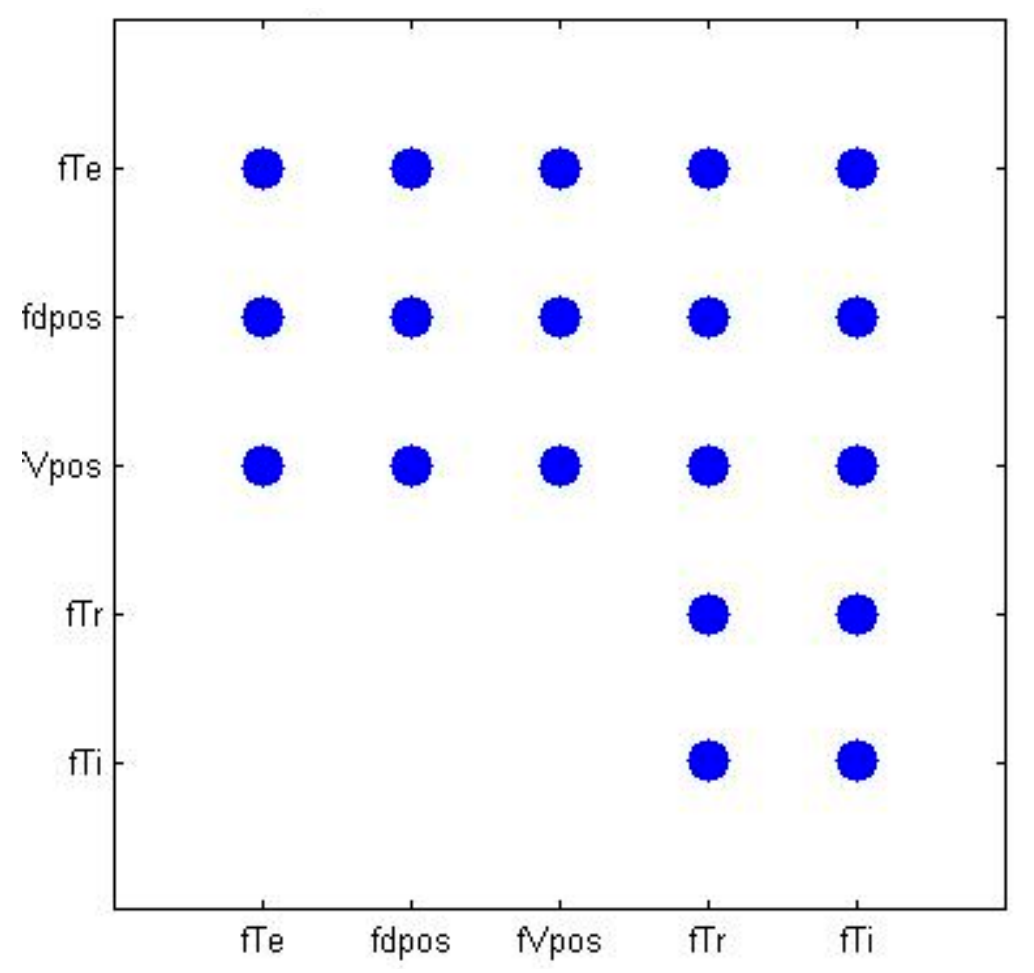

Figure 4. Fault isolability matrix for scenario 2.

For scenario 1, full isolability can be obtained by applying one of five identified solutions to placement of additional sensors. Each solution contains two sensors that must be added to the system. Among the identified solutions, it does not influence the isolability which of the solutions that are chosen. Thus, all the identified solutions ensure fully isolability. A solution could be to place sensors in $T_{r}$ and $T_{e}$, or in $T_{e}$ and $T_{\text {out }}$. However, full isolability is not attained if the sensors are paired differently, e.g., $T_{r}$ and $T_{\text {out }}$. As mentioned, there are three more solutions than these.

For scenario 2, the system is more complex as it contains more faults. Full isolability can be obtained by two set of solutions. Here, each solution requires three sensors to be added to the system. Sensors can be placed in $T_{e}, d_{p o s}$ and $V_{p o s}$, or in $T_{o u t}, d_{p o s}$ and $V_{p o s}$ to gain full isolability.

The results for scenario 2 with additional sensors can be seen in Figure 5. The result of the scenario is full isolability, where fault $i$ only occurs when it is present itself. Thereby, the number of faults occurring if a single fault is present has been drastically reduced. Now, it must be considered that there is also a possibility that one of the new added sensors might become faulty.

Again, the FDT can be used to foresee how such a faulty sensor influences the rest of the system. To illustrate this, scenario 2 is used as an example. Here, it is assumed that additional sensors have been added in the following placements: $T_{e}, d_{p o s}$ and $V_{p o s}$-as it can be seen in Figure 5 .

Now, a fault is introduced in the new added sensor $T_{e}$ which will be called fzTe. Figure 6 shows fzTe among the other faults in the fault isolability analysis. In this case, it can be seen that fzTe doesn't really influence the fault isolability of the system. This is due to the high level of isolability that already has been obtained by adding the three additional sensors. However, in some cases faulty new sensors might affect the isolability of the system. 


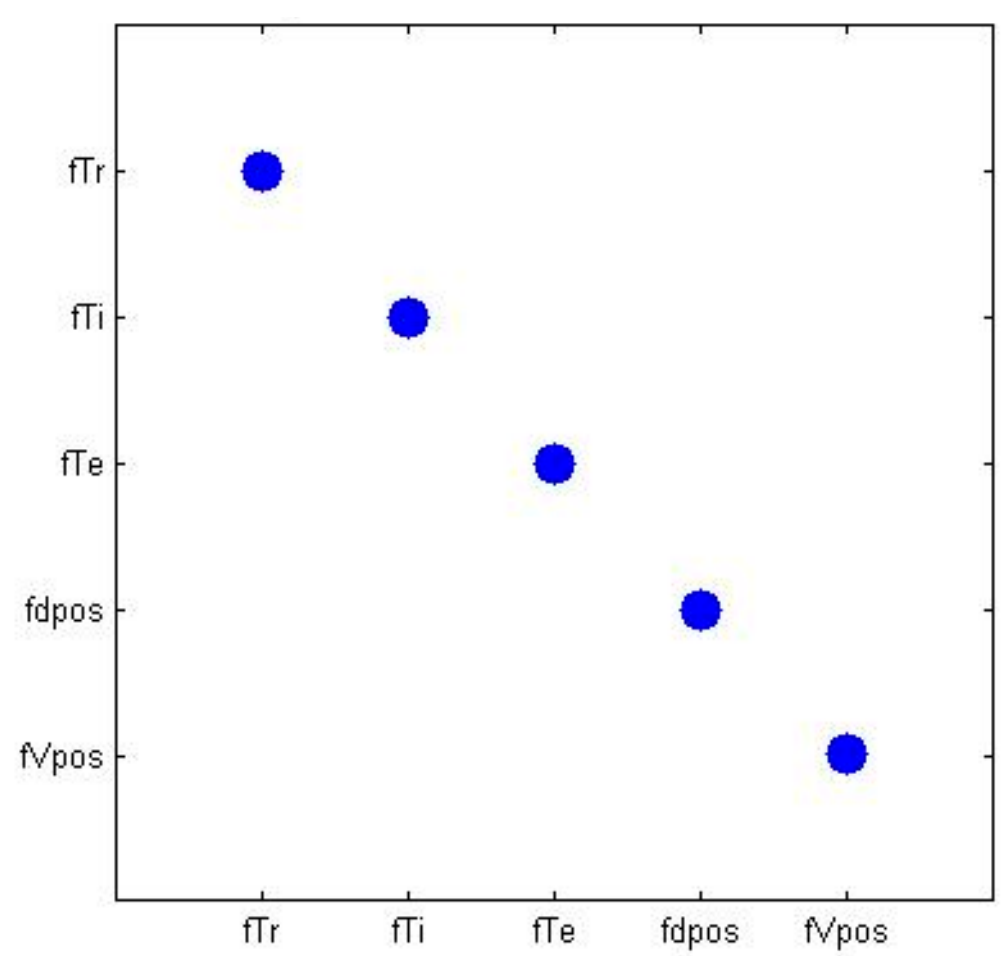

Figure 5. Isolability matrix for scenario 2 with additional sensors added. Fully isolability has been obtained by adding sensors at location $T_{e}, d_{p o s}$ and $V_{p o s}$.

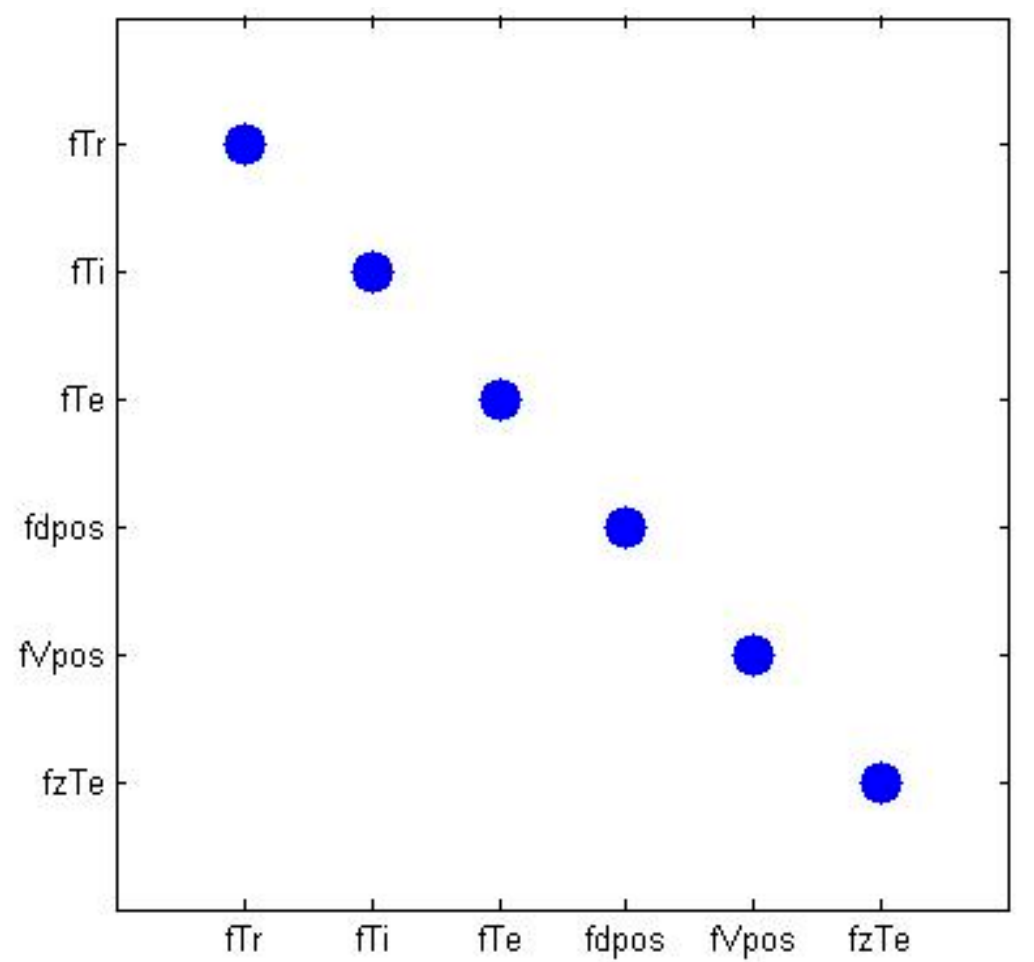

Figure 6. New added sensor Te has become faulty and fault fzTr has been added to the isolability matrix.

\subsection{Discussion}

There are different issues related to adding sensors to buildings for fault detection and diagnosis. When sensors are added to the system, it is important to investigate how the sensors are added to achieve the maximum benefits taking into account the limitations. Whether the aim is to ensure full detectability or full isolability of the faults, it requires different number of sensors and different sensor 
placements to achieve the goals. There is an important cost consideration that should be taken into account. Wireless sensor systems are expensive to add, and therefore the sensors must be added taking into account the return on investment. In large, complex models, fully isolability can require that many extra sensors are added. This can be expensive.

In general, it is not necessary required that the system acquires full isolability. The systems performance can easily be increased by adding just a few extra sensors. The trial and error approach used in this paper, works brilliantly to identify how sensors stepwise can improve the isolability. Here, the task will be to investigate in which positions the sensors will influence the system the most. It should also be taken into account that not all faults are equally important. Therefore, it could also be a question of ensuring isolability for the faults that causes the highest financial impact to the building.

As mentioned, operation faults such as incorrect HVAC on/off modes and inappropriate set points accounted for large energy wastes and thereby a large financial loss [8]. Therefore, there is a large potential and an obvious economic incentive to detect and diagnose faults. If wireless temperature sensors are added to rooms that were not previously equipped with sensors or where the sensors were faulty, energy savings can be attainted. Studies claim that the payback period for installing wireless temperature sensor networks in buildings can come down to a satisfactory 7 months [22].

\section{Conclusions}

This paper looked into the possibilities to use Dulmage-Mendelsohn decomposition for building applications. Along with the Dulmage-Mendelsohn decomposition, fault diagnosability analysis and sensor placement analysis were conducted. The methods were applied for the first time for application in smart buildings. The methods were tested on a room of the OU44 building which was equipped with sensors measuring temperatures and ventilation damper and heating valve positions. Based on the state-space model of the room of the OU44 building, the fault diagnosis was conducted. The study was divided into two scenarios which contained a different number and kinds of faults. The main findings of this study are described are as follows:

- It was well demonstrated that the methods could successfully be used for building applications. It was possible to do the fault diagnosis of the faults introduced to the model. Here, the method was capable of handling five faults (in scenario 2).

- Systems that contain underdetermined parts cannot be used in a sensor placement analysis. As the sensor placement algorithm looks for all sensor sets ensuring full isolability, the set of sensors becomes too big to handle. Therefore, scenarios, where different variables (e.g., valve positions) were assumed to be constant, were designed to avoid underdetermined parts of the system.

- Full detectability and isolability was obtained by adding additional sensors to the system by using the sensor placement algorithm. It was found that as the system gets more complex through introducing more faults, additional sensors should be added to gain full isolability.

This paper presented a method that can be used for fault diagnosability analysis for building applications. Further, there is a potential to use the method to design residual generators. Residual generators can be designed by using sets of equations from the model that contain redundancy. Here, knowledge about the redundancy identified in the Dulmage-Mendelnsohn decomposition is an important aspect. The designed residual generators can through a generated fault signal tells where and when a fault is present. Thus, residual generators can make the fault detection and diagnosis automatic. Nevertheless, it shall be highlighted that in addition to the large technical potential and the economic feasibility of applying the proposed approach, an automated fault detection and diagnosis process could majorly lead to less operational costs, less maintenance costs, and better time and resources allocation. 
Author Contributions: Conceptualization, M.E.S.T. and H.R.S.; Methodology, M.E.S.T. and H.R.S.; Formal Analysis, M.E.S.T., H.R.S., M.J. and K.A.; Investigation, M.E.S.T.; Resources, H.R.S., M.J. and K.A.; Writing Original Draft Preparation, M.E.S.T. and H.R.S.; Writing-Review \& Editing, M.E.S.T., H.R.S., M.J. and K.A.; Supervision, H.R.S., M.J. and K.A.; Funding Acquisition, H.R.S., M.J. and K.A.

Funding: This work is supported by the Innovation Fund Denmark for the project COORDICY.

Conflicts of Interest: The authors declare no conflict of interest.

\section{Nomenclature}

\begin{tabular}{|c|c|c|}
\hline Symbol & Description & Units \\
\hline$C_{e}$ & External thermal mass capacitance. & $\mathrm{J} / \mathrm{K}$ \\
\hline$C_{i}$ & Internal thermal mass capacitance. & $\mathrm{J} / \mathrm{K}$ \\
\hline$C_{r}$ & Thermal capacitance for the room. & $\mathrm{J} / \mathrm{K}$ \\
\hline$d_{p o s}$ & Ventilation damper position. & $\%$ \\
\hline FDD & Fault detection and diagnosis. & \\
\hline FDT & MatLab-driven Fault Diagnosis Toolbox & \\
\hline$f_{\text {dpos }}$ & Percentage fault in $d_{p o s}$ & $\%$ \\
\hline$f_{T e}$ & Sensor fault in $T_{e}$. & \\
\hline$f_{T i}$ & Sensor fault in $T_{i}$. & \\
\hline$f_{T r}$ & Sensor fault in $T_{r}$. & \\
\hline$f_{\text {sol }}$ & Solar coefficient. & \\
\hline$f_{V p o s}$ & Percentage fault in $V_{p o s}$ & $\%$ \\
\hline$f_{z T r}$ & Faulty new added sensor in $T_{r}$. & \\
\hline$H_{\text {glo }}$ & Horizontal global radiation. & W \\
\hline$q_{o c c}$ & Occupancy heat gain. & W \\
\hline$Q_{\text {rad }}$ & Maximum radiator power. & W \\
\hline$q_{V e}$ & Ventilation heat gain. & $\mathrm{W}$ \\
\hline$R_{e 1}$ & External wall resistance. & $\mathrm{K} / \mathrm{W}$ \\
\hline$R_{e 2}$ & External wall resistance & $\mathrm{K} / \mathrm{W}$ \\
\hline$R_{i}$ & Internal wall resistance & $\mathrm{K} / \mathrm{W}$ \\
\hline$\dot{T}_{e}$ & Time derivative of $\boldsymbol{T}_{\boldsymbol{e}}$ & ${ }^{\circ} \mathrm{C}$ \\
\hline$T_{e}$ & External thermal mass temperature. & ${ }^{\circ} \mathrm{C}$ \\
\hline$\dot{T}_{i}$ & Time derivative of $\boldsymbol{T}_{\boldsymbol{i}}$ & ${ }^{\circ} \mathrm{C}$ \\
\hline$T_{i}$ & Internal thermal mass temperature. & ${ }^{\circ} \mathrm{C}$ \\
\hline$T_{\text {out }}$ & Outdoor temperature. & ${ }^{\circ} \mathrm{C}$ \\
\hline$\dot{T}_{r}$ & Time derivative of $\boldsymbol{T}_{\boldsymbol{r}}$. & ${ }^{\circ} \mathrm{C}$ \\
\hline$T_{r}$ & Room temperature. & ${ }^{\circ} \mathrm{C}$ \\
\hline$T_{V e}$ & Ventilation air temperature. & ${ }^{\circ} \mathrm{C}$ \\
\hline$V_{\max }$ & Maximum airflow rate into the room when damper is fully open. & $\mathrm{m}^{3} / \mathrm{h}$ \\
\hline$V_{\text {pos }}$ & Radiator valve position. & $\%$ \\
\hline$y_{T e}$ & Sensor measurement of $T_{e}$. & ${ }^{\circ} \mathrm{C}$ \\
\hline$y_{T i}$ & Sensor measurement of $T_{i}$. & ${ }^{\circ} \mathrm{C}$ \\
\hline$y_{T r}$ & Sensor measurement of $T_{r}$. & ${ }^{\circ} \mathrm{C}$ \\
\hline$y_{q v e}$ & Sensor measurement of $q_{v e}$. & ${ }^{\circ} \mathrm{C}$ \\
\hline
\end{tabular}

\section{References}

1. European Commision. Building Energy Consumption in the EU. 2018. Available online: https://ec.europa. eu/energy/en/topics/energy-efficiency/buildings (accessed on 24 January 2019).

2. Allouhi, A.; El Fouih, Y.; Kousksou, T.; Jamil, A.; Zeraouli, Y.; Mourad, Y. Energy consumption and efficiency in buildings: current status and future trends. J. Clean. Prod. 2015, 109, 118-130. [CrossRef]

3. Lu, J.; Sookoor, T.; Srinivasan, V.; Gao, G.; Holben, B.; Stankovic, J.; Field, E.; Whitehouse, K. The smart thermostat: using occupancy sensors to save energy in homes. In Proceedings of the 8th ACM Conference on Embedded Networked Sensor Systems, Zürich, Switzerland, 3-5 November 2010; pp. 211-224. [CrossRef] 
4. Pérez-Lombard, L.; Ortiz, J.; Pout, C. A review on buildings energy consumption information. Energy Build. 2008, 40, 394-398.

5. Jagpal, R. Computer-Aided Evaluation of HVAC System Performance. Technical Report. IEA, 2002. Available online: http://www.iea-ebc.org/Data/publications/EBC_Annex_34_tsr.pdf (accessed on 24 January 2019).

6. EECCAC. Project for the Directorate General Transportation-Energy of the Commission of the European Union. Final Report. April 2003. Available online: https://lms.i-know.com/pluginfile.php/28688/mod_resource/ content/57/Energy\%20Efficiency\%20and\%20Certification\%20of\%20Central\%20Air\%20Conditioners.pdf (accessed on 24 January 2019).

7. Russell, E.; Chiang, L.; Braatz, R. Data-Driven Methods for Fault Detection and Diagnosis in Chemical Processes; Springer: New York, NY, USA, 2012; ISBN 978-1-4471-0409-4.

8. Kim, J.; Cai, J.; Braun, J.E. Common Faults and Their Prioritization in Small Commercial Buildings: February 2017-December 2017; Department of Energy-National Renewable Energy Laboratory: Golden, CO, USA, 2018. Available online: https://www.nrel.gov/docs/fy18osti/70136.pdf (accessed on 24 January 2019).

9. Sorrentino, M.; Rizzo, G.; Trifiro, A.; Bedogni, F. A model-based key performance index for energy assessment and monitoring of telecommunication cooling systems. IEEE Trans. Sustain. Energy 2014, 5, 1126-1136. [CrossRef]

10. Polverino, P.; Sorrentino, M.; Pianese, C. A model-based diagnostic technique to enhance faults isolability in Solid Oxide Fuel Cell systems. Appl. Energy 2017, 204, 1198-1214. [CrossRef]

11. Katipamula, S.; Brambley, M.R. Methods for Fault Detection, Dianostics, and Prognostics for Building Systems-A Review, Part I. HVACER Res. 2005, 11, 3-25.

12. Hong, T.; Zhang, R. Modeling of HVAC operational faults in building performance simulation. Appl. Energy 2017, 202, 178-188.

13. Kim, W.; Katipamula, S. A Review of Fault Detection and Diagnostics Methods for Building Systems. Sci. Technol. Built Environ. 2018, 24, 3-21. [CrossRef]

14. Frisk, E.; Krysander, M.; Jung, D. A Toolbox for Analysis and Design of Model Based Diagnosis Systems for Large Scale Models. IFAC-PapersOnLine 2017, 50, 3287-3293. [CrossRef]

15. Krysander, M.; Frisk, E. Sensor Placement for Fault Diagnosis. IEEE Trans. Syst. Man Cybern. Part A Syst. Humans 2008, 38, 1398-1410. [CrossRef]

16. Arendt, K.; Jradi, M.; Shaker, H.; Veje, C. Comparative Analysis of White-, Grey, and Black-box Models for Thermal Simulation of Indoor Environment: Teaching Building Case Study. In Proceedings of the 2018 Building Performance Modeling Conference and SimBuild co-organized by ASHRAE and IBPSA-USA, Chicago, IL, USA, 26-28 September 2018.

17. Koscielny, J.M.; Syfert, M.; Rostek, K.; Sztyber, A. Fault Isolability with different Forms of the Faults-Symptoms Relation. Appl. Math. Comput. 2016, 26, 815-826. [CrossRef]

18. Bang, M.; Engelsgaard, S.S.; Andersen, E.K.; Skydt, M.R.; Shaker, H.R.; Jradi, M. Novel real-time model-based fault detection method for automatic identification of abnormal energy performance in building ventilation units. Energy. Build. 2019, 183, 238-251. [CrossRef]

19. Bynum, J.D.; Claridge, D.E.; Curtin, J.M. Development and testing of an automated building commissioning analysis tool (abcat). Energy Build 2012, 55, 607-617. [CrossRef]

20. Eschevarria, L.C.; de Campos Vehlo, H.F.; Becceneri, J.C.; da Silva Neto, A.J.; Santiago, O.L. The fault diagnosis inverse problem with Ant Colony Optimization and Ant Colony Optimization with dispersion. Appl. Math. Comput. 2014, 227, 687-700.

21. Frisk, E. Fault Diagnosis Toolbox—v0.13, Department of Electrical Engineering; Linköping University: Linköping, Sweden, 2018.

22. Capehart, B.; Middelkoop, T. Handbook of Web Based Energy Information and Control Systems; The Fairmont Press: Atlanta, GA, USA, 2011; pp. 478-481.

(C) 2019 by the authors. Licensee MDPI, Basel, Switzerland. This article is an open access article distributed under the terms and conditions of the Creative Commons Attribution (CC BY) license (http://creativecommons.org/licenses/by/4.0/). 